\title{
Perfil microbiológico das culturas de pacientes internados na Sala de Cuidados Intermediários de um Hospital Universitário
}

\author{
Microbiological profile of cultures from patients admitted to the Intermediate Care Room of a \\ University Hospital \\ Perfil microbiológico de cultivos de pacientes ingresados en la Sala de Cuidados Intermedios de un \\ Hospital Universitario
}

Recebido: 15/07/2021 | Revisado: 20/07/2021 | Aceito: 21/07/2021 | Publicado: 28/07/2021

\author{
Cleusa Wanderley de Queiroz Andrade \\ ORCID: https://orcid.org/0000-0001-6113-1432 \\ Universidade Federal do Vale do São Francisco, Brasil \\ E-mail: cleusawqandrade@gmail.com \\ Katia Suely Batista Silva \\ ORCID: https://orcid.org/0000-0001-6146-4229 \\ Universidade Federal do Vale do São Francisco, Brasil \\ E-mail: katiasuelybs@gmail.com \\ Mirthes Maria Rodrigues Santana \\ ORCID: https://orcid.org/0000-0002-7087-7145 \\ Universidade Federal do Vale do São Francisco, Brasil \\ E-mail: Mirthes-mari@hotmail.com \\ Aline Vitória de Oliveira \\ ORCID: https://orcid.org/0000-0002-3308-455X \\ Centro Universitário Barão de Mauá, Brasil \\ E-mail: alineovitoria@gmail.com \\ Marcos Duarte Guimarães \\ ORCID: https://orcid.org/0000-0002-9458-5649 \\ Universidade Federal do Vale do São Francisco, Brasil \\ E-mail: marcosduarte500@gmail.com \\ Carine Rosa Naue \\ ORCID: https://orcid.org/0000-0003-4215-3606 \\ Universidade Federal do Vale do São Francisco, Brasil \\ E-mail: crnaue@yahoo.com.br
}

\begin{abstract}
Resumo
Avaliar o perfil bacteriano das amostras de aspirados traqueais e uroculturas de pacientes internados na Sala de Cuidados Intermediários do Hospital Universitário do Vale do São Francisco, Petrolina/PE. Trata-se de um estudo descritivo, quantitativo e retrospectivo envolvendo a análise do perfil microbiológico das amostras coletadas no período de janeiro a dezembro de 2020 pelo Laboratório de Análises Clínicas do Hospital Universitário. Os dados foram tabulados em planilhas do Excelß, sendo feito a análise descritiva com valores percentuais e absolutos. As identificações bacterianas e os antibiogramas foram realizados através do sistema automatizado BD Phoenix TM, seguindo a metodologia do Clinical and Laboratory Standards Institute. Foram coletados 120 aspirados traqueais, sendo 55 positivos (46\%) para achados bacterianos; os patógenos mais prevalentes foram: Acinetobacter baumannii (40\%), Klebsiella pneumoniae (16\%) e Pseudomonas aeruginosa (11\%). Em relação às uroculturas, foram realizadas 183, sendo $10(5,46 \%)$ positivas para achados bacterianos; as bactérias mais prevalentes foram: Escherichia coli (40\%) e Enterobacter cloacae (20\%). A. baumannii apresentou 100\% de sensibilidade à colistina e polimixina B nos aspirados traqueias, assim como a K. pneumoniae apresentou à amicacina em todas as amostras coletadas. A E. coli demonstrou certa restência às cefalospororinas, com exceção da cefoxitina, sendo sensível; além disso, teve sensibilidade parcial ao sulfametoxazol+trimetoprima no estudo. O conhecimento do perfil microbiológico das bactérias permite a elaboração de protocolos preventivos e a realização de tratamento efetivos, diminuindo as taxas de mortalidade, o tempo de internação e os custos em saúde.
\end{abstract}

Palavras-chave: Infecção hospitalar; Bactérias; Resistência bacteriana a antibióticos; Microbiologia; Hospitais universitários.

\footnotetext{
Abstract

Evaluate the bacterial profile of samples of tracheal aspirates and urine cultures from patients admitted to the Intermediate Care Room of the University Hospital of Vale do São Francisco, Petrolina/PE. This is a descriptive, quantitative and retrospective study involving the analysis of the microbiological profile of samples collected from
} 
January to December 2020 by the Clinical Analysis Laboratory of the University Hospital. Data were tabulated in Excel ${ }^{\circledR}$ spreadsheets, where descriptive analysis was performed with absolute and percentage values. Bacterial identifications and antibiograms were performed using the BD Phoenix ${ }^{\mathrm{TM}}$ automated system, following the Clinical and Laboratory Standards Institute methodology. 120 tracheal aspirates were collected, being 55 positive (46\%) for bacterial findings; the most prevalent pathogens were: Acinetobacter baumannii (40\%), Klebsiella pneumoniae (16\%) and Pseudomonas aeruginosa (11\%). Regarding urine cultures, 183 were performed, $10(5.46 \%)$ of which were positive for bacterial findings; the most prevalent bacteria were: Escherichia coli $(40 \%)$ and Enterobacter cloacae (20\%). A. baumannii showed $100 \%$ sensitivity to colistin and polymyxin B in tracheal aspirates, as well as $K$. pneumoniae to amikacin in all collected samples. E. coli showed some cephalospororin restriction, with the exception of cefoxitin, being sensitive; in addition, he had partial sensitivity to sulfamethoxazole+trimethoprim in the study. Knowledge of the microbiological profile of bacteria allows the development of preventive protocols and effective treatment, reducing mortality rates, length of stay and health care costs.

Keywords: Cross infection; Bacteria; Drug resistance, Bacterial; Microbiology; Hospitals, university.

\section{Resumen}

Evaluar el perfil bacteriano de muestras de aspirados traqueales y urocultivos de pacientes ingresados en la Sala de Cuidados Intermedios del Hospital Universitario Vale do São Francisco, Petrolina / PE. Se trata de un estudio descriptivo, cuantitativo y retrospectivo que implica el análisis del perfil microbiológico de muestras recolectadas de enero a diciembre de 2020 por el Laboratorio de Análisis Clínicos del Hospital Universitario. Los datos se tabularon en hojas de cálculo Excel®, donde se realizó un análisis descriptivo con valores absolutos y porcentuales. Se realizaron identificaciones bacterianas y antibiogramas utilizando el sistema automatizado BD Phoenix ${ }^{\mathrm{TM}}$, siguiendo la metodología del Clinical and Laboratory Standards Institute. Se recolectaron 120 aspirados traqueales, siendo 55 positivos (46\%) para hallazgos bacterianos; los patógenos más prevalentes fueron: Acinetobacter baumannii (40\%), Klebsiella pneumoniae (16\%) y Pseudomonas aeruginosa (11\%). En cuanto a urocultivos, se realizaron 183, de los cuales $10(5,46 \%)$ resultaron positivos para hallazgos bacterianos; las bacterias más prevalentes fueron: Escherichia coli $(40 \%)$ y Enterobacter cloacae (20\%). A. baumannii mostró una sensibilidad del $100 \%$ a la colistina y polimixina $\mathrm{B}$ en los aspirados traqueales, así como $K$. pneumoniae a la amikacina en todas las muestras recolectadas. E. coli mostró cierta restricción de cefalospororina, con la excepción de cefoxitina, que es sensible; además, tenía sensibilidad parcial al sulfametoxazol + trimetoprima en el estudio. El conocimiento del perfil microbiológico de las bacterias permite el desarrollo de protocolos preventivos y de tratamiento eficaz, reduciendo las tasas de mortalidad, la duración de la estancia y los costes sanitarios.

Palabras clave: Infección hospitalaria; Bacterias; Farmacorresistencia bacteriana; Microbiología; Hospitales universitarios.

\section{Introdução}

O Ministério da Saúde e a Agência Nacional de Vigilância Sanitária (ANVISA) substituíram o termo Infecções Hospitalares $(\mathrm{IH})$ por Infecções Relacionadas à Assistência à Saúde (IRAS). Essa nova denominação abrange as infecções adquiridas em qualquer ambiente que preste assistência à saúde, seja atendimento hospitalar, ambulatorial, domiciliar ou infecções adquiridas após alta.

As IRAS se destacam cada vez mais na área médica, devido sua fácil e ampla disseminação, causando grande impacto nos serviços de saúde e na população (Gomes \& Moraes, 2018). No ambiente hospitalar, as IRAS estão relacionadas principalmente a procedimentos realizados durante a internação. As Unidades de Cuidados Intermediários (UCI) são ambientes de assistência multiprofissional contínua a pacientes de risco moderado ou semicríticos. Nesses locais de cuidado há um risco de infecção por patógenos pelos pacientes, já que há uma constante exposição a procedimentos invasivos (Sousa et al., 2017).

Em uma revisão integrativa realizada por Sousa et al, foi demostrado que a natureza do procedimento, o ambiente, a técnica e o tempo de permanência do cateter foram descritos como os principais fatores de risco para o desenvolvimento das IRAS. Observou-se também que os principais dispositivos relacionados com o desenvolvimento de infecções são a cateterização urinária, intubação endotraqueal, ventilação mecânica e cateteres intravasculares (Sousa et al., 2017).

A ANVISA desenvolve estudos sobre critérios diagnósticos das infecções mais frequentes relacionadas aos serviços de saúde desde 2008. Nesses estudos, foi possível observar que as infecções mais prevalentes entre as IRAS são as pneumonias relacionadas à ventilação mecânica, as infecções hematológicas, as infecções do trato geniturinário relacionadas a dispositivos invasivos e as infecções de sítio cirúrgico (Agência Nacional de Vigilância Sanitária, 2017). 
Entre os microrganismos mais prevalentes em hemoculturas, destacam-se os cocos gram-positivos Staphylococcus sp., sendo em sua grande maioria resistentes à oxacilina, ampicilina e sulfazotrim e, quanto aos gram-negativos, a Escherichia coli, a Enterobacter sp. e a Klebsiella sp., possuindo resistências variáveis as fluoroquinolonas, monobactâmicos, cefalosporinas de $3^{\mathrm{a}}$ geração, ampicilina e ao ácido clavulânico (Oliveira \& Santos, 2020).

O crescente aumento da resistência bacteriana gera preocupação, pois influencia diretamente no prolongamento das internações, na oneração dos serviços de saúde e aumenta o risco de desenvolvimento de bactérias multirresistentes. Dessa forma, o maior problema relacionado às IRAS é o surgimento da multirresistência bacteriana, que está associado ao uso indiscriminado dos antibióticos, dose e tempo inadequado, além da falta de treinamento apropriado dos profissionais de saúde (Ribeiro et al., 2019; Andrade et al., 202; Agência Nacional de Vigilância Sanitária, 2016).

Dentro desse contexto, o conhecimento do perfil bacteriano pode direcionar o tratamento dessas infecções e, assim, prevenir e controlar as IRAS. Assim, o objetivo deste trabalho consiste em estudar o perfil microbiológico dos aspirados traqueais e das uroculturas coletados de pacientes internados na Sala de Cuidados Intermediários (SCI) do Hospital Universitário de Petrolina, Pernambuco.

\section{Metodologia}

A pesquisa foi realizada no Hospital Universitário da Universidade Federal do Vale do São Francisco (HUUNIVASF/EBSERH), localizado em Petrolina/PE. O hospital possui perfil assistencial de hospital geral de média e alta complexidade à população adulta, sendo referência em traumas, politraumas, ortopedia, neurocirurgia, clínica geral e médica para a VIII Gerência Regional de Saúde (GERES) de Pernambuco. Dessa forma, o hospital comporta a população dos municípios de Afrânio, Cabrobó, Dormentes, Lagoa Grande, Orocó, Petrolina e Santa Maria da Boa Vista; o que corresponde a 475.740 habitantes (Secretaria Estadual de Saúde de Pernambuco, 2020).

Trata-se de um estudo descritivo, quantitativo e retrospectivo realizado a partir da coleta de dados dos aspirados traqueais e das uroculturas coletadas de pacientes internados na Sala de Cuidados Intermediários no período de janeiro a dezembro de 2020, disponibilizados pelo laboratório de Análises Clínicas e Anatomia Patológica do hospital.

Os resultados dos exames foram tabulados em planilhas do Excel ${ }^{\circledR}$ e foram divididos em amostras positivas e negativas. Após isso, foi realizada a análise descritiva com valores percentuais e absolutos. As amostras de urocultura com contagem de colônias igual ou superior a $100.000 \mathrm{UFC} / \mathrm{mL}$ foram consideradas positivas; enquanto, para as culturas de aspirados traqueias, foram consideradas positivas quando houve contagem de colônias igual ou superior a $10^{6} \mathrm{UFC} / \mathrm{mL}$.

Nas amostras positivas foram analisadas as variáveis ocorrência de bactérias e o perfil de sensibilidade e resistência aos antibióticos testados. Os dados foram organizados e apresentados por meio de gráficos e tabelas construídos no programa Excel® para demonstração dos resultados.

Para a identificação bacteriana e dos antibiogramas foi utilizado o sistema automatizado PHOENIX, da BD, com os painéis adequados. Os resultados foram classificados em sensível (S) e resistente (R), seguindo a metodologia do Clinical and Laboratory Standards Institute (2018).

A pesquisa foi submetida e aprovada no Comitê de Ética da Universidade Federal Rural de Pernambuco (UFRPE), através do CAAE: 40360120.4.0000.9547.

\section{Resultados}

Foram coletados 120 aspirados traqueais, sendo 55 positivos (46\%) e 65 negativos (54\%) para achados bacterianos. Foram confirmados 12 agentes etiológicos nos aspirados traqueais: Acinetobacter baumannii, Klebsiella pneumoniae, Pseudomonas aeruginosa, Staphylococcus aureus, Corynebacterium striatum, Escherichia coli, Enterobacter cloacae, Serratia 
marcescens, Burkholderia cepacia complexo, Enterococcus faecalis, Providencia rettgeri e Proteus mirabilis. As bactérias mais prevalentes foram Acinetobacter baumannii (40\%), Klebsiella pneumoniae (16\%) e Pseudomonas aeruginosa (11\%); as demais bactérias correspondem a 33\% dos casos (Gráfico 1).

Gráfico 1. Ocorrência de bactérias isoladas em aspirados traqueais coletadas em pacientes internados na SCI do HUUNIVASF (EBSERH) no período de janeiro a dezembro de 2020.

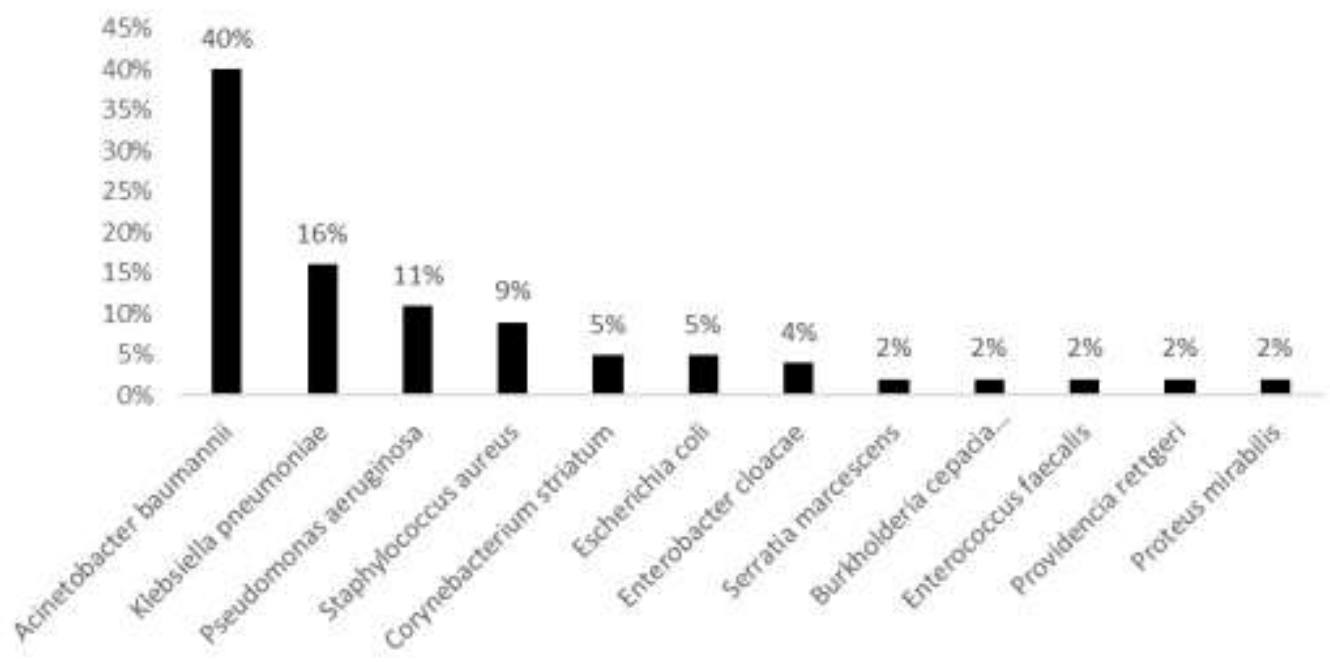

Fonte: Autores (2021).

Em relação às uroculturas, foram realizadas 183 , sendo 10 positivas para achados bacterianos, 12 para fungos e 161 negativas. A incidência dos fungos foi de 6 casos para Cândida tropicalis, 5 para Cândida albicans e 1 para Cândida glabatra. Foram encontrados os seguintes agentes etiológicos quanto à pesquisa por bactérias: Escherichia coli, Enterobacter cloacae, Enterococcus faecium, Staphylococcus haemolyticus, Klebsiella pneumoniae e Pseudomonas aeruginosa. Observa-se no Gráfico 2 que as espécies bacterianas mais frequentes foram Escherichia coli (40\%), Enterobacter cloacae (20\%) e Enterococcus faecalis (10\%).

Gráfico 2. Ocorrência de bactérias isoladas em uroculturas coletadas em pacientes internados na SCI do HU-UNIVASF (EBSERH) no período de janeiro a dezembro de 2020.

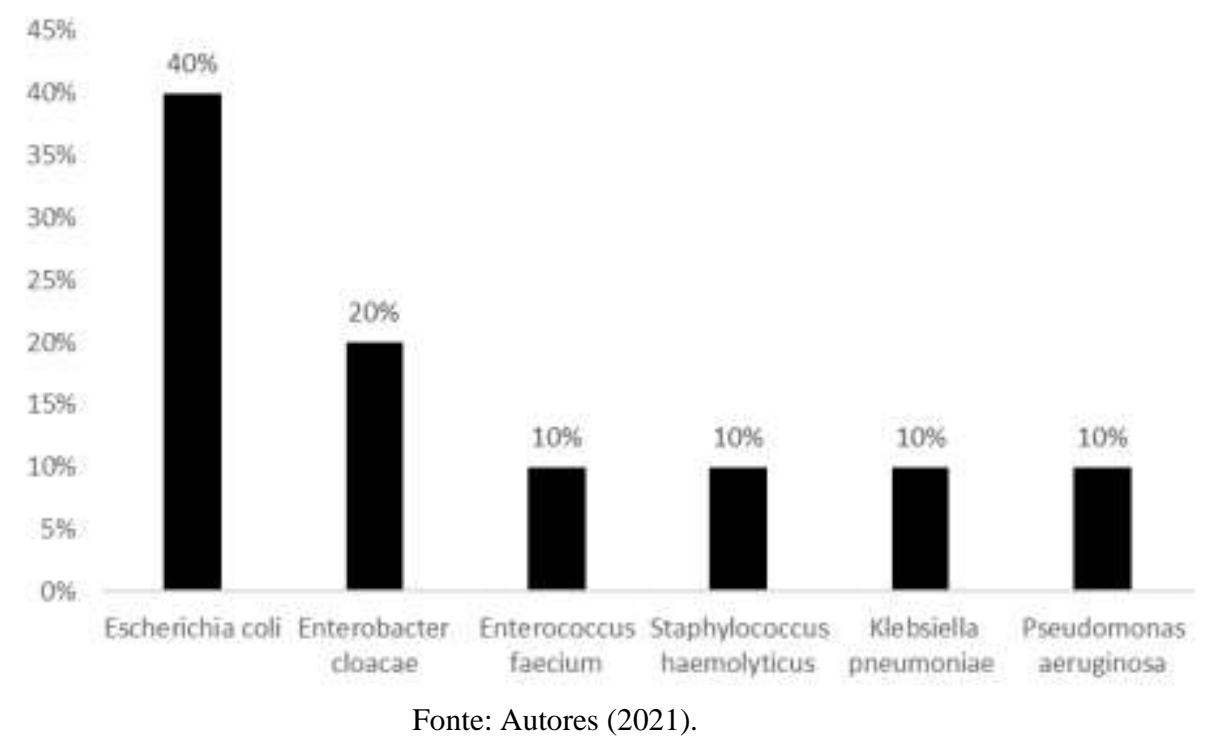


Encontrou-se nos aspirados traqueais as seguintes bactérias gram-positivas: 5 casos de Staphylococcus aureus, 3 de Corynebacterium striatum e 1 de Enterococcus faecalis.

O Staphylococcus aureus apresentou resistência a clindamicina (20\%), oxacilina (20\%) e 100\% de sensibilidade a sulfametoxazol + trimetoprima e vancomicina.

Não foi realizado o perfil de resistência da Corynebacterium striatum porque não existem antibióticos padronizados pela Clinical and Laboratory Standards Institute para este agente etiológico.

Para Enterococcus faecalis foi observado $100 \%$ de resistência apenas a ceftarolina e $100 \%$ de sensibilidade a ampicilina, linezolide, penicilina, tigeciclina e vancomicina.

Sobre o perfil de resistência da Acinetobacter baumannii, não houve $100 \%$ de resistência a nenhum dos antibióticos testados; houve resistência variável a ampicilina + sulbactam (41\%), imipinem (95\%), meropenem (95\%) e 100\% de sensibilidade a polimixina B e colistina (Tabela 1).

Os isolados de Klebsiella pneumoniae apresentaram os percentuais de resistência de $11 \%$ a imipinem, $11 \%$ a meropenem e $33 \%$ a piperacilina + tazobactam. Todos os isolados foram $100 \%$ sensíveis a amicacina (Tabela 1).

Para Pseudomonas aeruginosa, verificou-se 100\% de resistência a todos os antimicrobianos testados: amicacina, cefepime, ceftazidina, ciprofloxacino, imipinem, levofloxacino, meropenem e piperacilina + tazobactam (Tabela 1).

Em relação aos isolados de Escherichia coli, verificou-se os seguintes percentuais de resistência (Tabela 1): 100\% a ampicilina e levofloxacino, $67 \%$ a ampicilina + sulbactam, 33\% a cefepime, 33\% a ceftriaxona, 33\% a ciprofloxacino, 33\% a gentamicina e $33 \%$ a sulfametoxazol + trimetoprima. Houve $100 \%$ de sensibilidade a amicacina, cefoxtina, ertapenem, imipinem, meropenem, piperacilina + tazobactam e tigeciclina.

Já a Enterobacter cloacae apresentou $100 \%$ de resistência a ampicilina, bem como a ampicilina + sulbactam, cefazolina, cefoxtina, ceftriaxona, ertapenem e piperacilina + tazobactam; resistência variável a cefepime (50\%), meropenem $(50 \%)$ e tigeciclina (50\%) e $100 \%$ de sensibilidade a amicacina, ciprofloxacino, imipinem e sulfametoxazol + trimetoprima (Tabela 1).

O caráter de resistência da Serratia marcescens observado foi de $100 \%$ de resistência a ampicilina, ampicilina + sulbactam, cefazolina, cefoxtina, ertapenem e tigeciclina e $100 \%$ de sensibilidade a amicacina, cefepime, ceftriaxona, ciprofloxacino, gentamicina, imipinem, meropenem, piperacilina + tazobactam e sulfametoxazol + trimetoprima.

Verificou-se que o isolado de Burkholderia cepacia complexo teve $100 \%$ de resistência a cefepime, ertapenem e imipinem e $100 \%$ de sensibilidade a ceftazidina, levofloxacino, meropenem e sulfametoxazol + trimetoprima.

O isolado de Providencia rettgeri apresentou $100 \%$ de resistência a ampicilina, ampicilina + sulbactam, cefazolina e tigeciclina e 100\% de sensibilidade aos seguintes antimicrobianos: amicacina, cefepime, cefoxtina, ceftriaxona, ciprofloxacino, ertapenem, gentamicina, imipinem, meropenem, piperacilina + tazobactam e sulfametoxazol + trimetoprima.

O agente etiológico Proteus mirabilis demonstrou aos antibióticos ertapenem e tigeciclina 100\% de resistência e $100 \%$ de sensibilidade a ampicilina, amicacina, ampicilina + sulbactam, cefepime, cefoxtina, ceftriaxona, gentamicina, meropenem, piperacilina + tazobactam e sulfametoxazol + trimetoprima. 
Tabela 1. Perfil de resistência das bactérias mais prevalentes gram-negativas isoladas de aspirados traqueais coletados de pacientes internados na SCI do HU-UNIVASF (EBSERH), no período de janeiro a dezembro de 2020.
A.baumannii
K. pneumoniae
P. aeruginosa
E. coli
E. cloacae

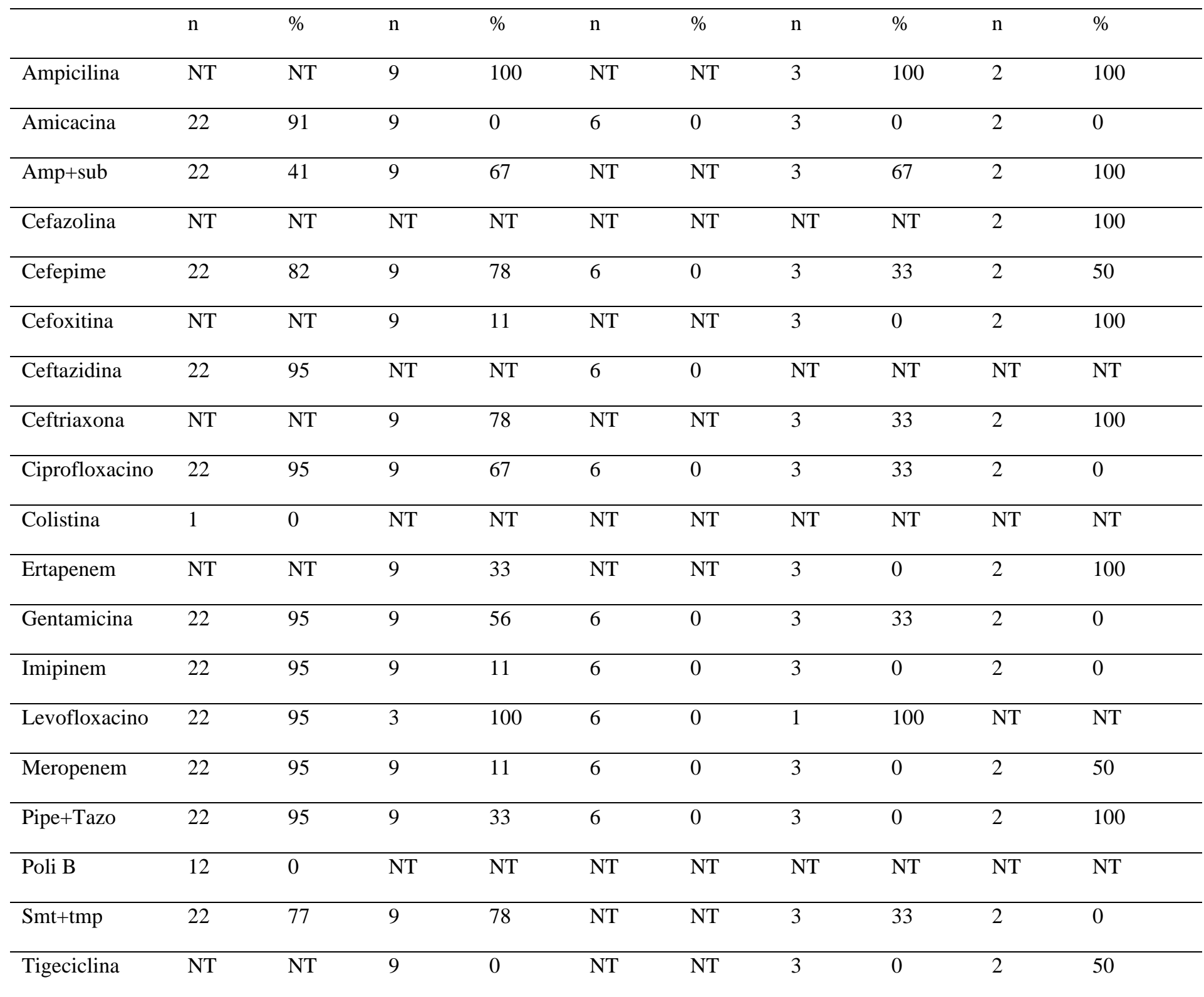

Legenda: NT: não testado; n: número de isolados bacterianos; \%: percentual de resistência; pipe+tazo: piperacilina + tazobactam; smt+tmp: sulfametoxazol + trimetoprima; amp+sub: ampicilina + sulbactam. Fonte: Autores (2021).

Em relação às uroculturas, o perfil de resistência encontrado no isolado de Enterococcus faecium foi de $100 \%$ de resistência a ampicilina e ciprofloxacino, enquanto houve $100 \%$ de sensibilidade a daptomicina, linezolide, nitroforuntoina, penicilina e vancomicina (Tabela 2).

Já o isolado de Staphylococcus haemolyticus apresentou $100 \%$ de resistência a ampicilina e penicilina e $100 \%$ de 
sensibilidade a daptomicina, linezolide, nitroforuntoina, oxacilina, rifampicina, sulfametoxazol + trimetoprima e vancomicina (Tabela 2).

A Escherichia coli apresentou $100 \%$ de resistência apenas ao antimicrobiano levofloxacino; resistência variável a ampicilina (50\%), cefazolina (25\%), cefepime (25\%), ceftriaxona (25\%) e ciprofloxacino (25\%); e $100 \%$ de sensibilidade a amicacina, ampicilina + sulbactam, cefoxitina, ertapenem, fosfomicina, gentamicina, imipinem, meropenem, piperacilina + tazobactam, sulfametoxazol + trimetoprima e tigeciclina (Tabela 2).

Os isolados de Enterobacter cloacae apresentaram $100 \%$ de resistência a ampicilina, ampicilina + sulbactam, cefazolina, cefepime, cefoxitina, ceftriaxona, ciprofloxacino e sulfametoxazol + trimetoprima. Foi observado $100 \%$ de sensibilidade a amicacina, ertapenem, gentamicina, imipinem, meropenem, piperacilina + tazobactam e tigeciclina (Tabela 2).

A Klebsiella pneumoniae exibiu $100 \%$ de sensibilidade a amicacina, imipinem, meropenem, piperacilina + tazobactam (Tabela 2).

Verificou-se que o isolado de Pseudomonas aeruginosa exibiu 100\% de sensibilidade a todos os antibióticos testados: amicacina, cefepime, ceftazidina, ciprofloxacino, imipinem, levofloxacino e piperacilina + tazobactam (Tabela 2). 
Tabela 2. Perfil de resistência das bactérias mais prevalentes isoladas em uroculturas de pacientes internados na SCI do HUUNIVASF (EBSERH), no período de janeiro a dezembro de 2020.

\section{Antimicronianos}

Espécies bacterianas

\begin{tabular}{|c|c|c|c|c|c|c|c|c|c|c|c|c|}
\hline \multirow[t]{2}{*}{ E. coli } & \multicolumn{2}{|c|}{ E. cloacae } & \multicolumn{2}{|c|}{ E. faecium } & \multicolumn{2}{|c|}{ S. haemolyticus } & \multicolumn{2}{|c|}{ K. pneumoniae } & \multicolumn{2}{|c|}{ P. aeruginosa } & \multirow[b]{2}{*}{$\mathrm{n}$} & \multirow[b]{2}{*}{$\%$} \\
\hline & $\mathrm{n}$ & $\%$ & $\mathrm{n}$ & $\%$ & $\mathrm{n}$ & $\%$ & $\mathrm{n}$ & $\%$ & $\mathrm{n}$ & $\%$ & & \\
\hline Ampicilina & 4 & 50 & 2 & 100 & 1 & 100 & 1 & 100 & 1 & 100 & NT & NT \\
\hline Amicacina & 4 & 0 & 2 & 0 & NT & NT & NT & NT & 1 & 0 & 1 & 0 \\
\hline Amp+sub & 4 & 0 & 2 & 100 & NT & NT & NT & NT & 1 & 0 & NT & NT \\
\hline Cefazolina & 4 & 25 & 2 & 100 & NT & NT & NT & NT & 1 & 0 & NT & NT \\
\hline Cefepime & 4 & 25 & 2 & 100 & NT & NT & NT & NT & 1 & 0 & 1 & 0 \\
\hline Cefoxitina & 4 & 0 & 2 & 100 & NT & NT & NT & NT & 1 & 0 & NT & NT \\
\hline Ceftazidina & NT & NT & NT & NT & NT & NT & NT & NT & NT & NT & 1 & 0 \\
\hline Ceftriaxona & 4 & 25 & 2 & 100 & NT & NT & NT & NT & 1 & 0 & NT & NT \\
\hline Ciprofloxacino & 4 & 25 & 2 & 100 & 1 & 100 & NT & NT & 1 & 0 & 1 & 0 \\
\hline Daptomicina & NT & NT & NT & NT & 1 & 0 & 1 & 0 & NT & NT & NT & NT \\
\hline Ertapenem & 4 & 0 & 2 & 0 & NT & NT & NT & NT & 1 & 0 & NT & NT \\
\hline Fosfomicina & 4 & 0 & NT & NT & NT & NT & NT & NT & NT & NT & NT & NT \\
\hline Gentamicina & 4 & 0 & 2 & 0 & NT & NT & NT & NT & 1 & 0 & 1 & 0 \\
\hline Imipinem & 4 & 0 & 2 & 0 & NT & NT & NT & NT & 1 & 0 & 1 & 0 \\
\hline Levofloxacino & 1 & 100 & NT & NT & NT & NT & NT & NT & NT & NT & 1 & 0 \\
\hline Linezolide & NT & NT & NT & NT & 1 & 0 & 1 & 0 & NT & NT & NT & NT \\
\hline Meropenem & 4 & 0 & 2 & 0 & NT & NT & NT & NT & 1 & 0 & NT & NT \\
\hline Nitrofurantoina & NT & NT & NT & NT & 1 & 0 & 1 & 0 & NT & NT & NT & NT \\
\hline Oxacilina & NT & NT & NT & NT & NT & NT & 1 & 0 & NT & NT & NT & NT \\
\hline Penicilina & NT & NT & NT & NT & 1 & 0 & 1 & 100 & NT & NT & NT & NT \\
\hline Pipe+Tazo & 3 & 0 & 2 & 0 & NT & NT & NT & NT & 1 & 0 & 1 & 0 \\
\hline Rifampicina & NT & NT & NT & NT & NT & NT & 1 & 0 & NT & NT & NT & NT \\
\hline Smt+tmp & 3 & 0 & 2 & 100 & NT & NT & 1 & 0 & 1 & 0 & NT & NT \\
\hline Tigeciclina & 3 & 0 & 2 & 0 & NT & NT & NT & NT & 1 & 0 & NT & NT \\
\hline Vancomicina & NT & NT & NT & NT & 1 & 0 & 1 & 0 & NT & NT & NT & NT \\
\hline
\end{tabular}

Legenda: NT: não testado; n: número de isolados bacterianos; \%: percentual de resistência; pipe+tazo: piperacilina + tazobactam; smt+tmp: sulfametoxazol + trimetoprima; amp+sub: ampicilina + sulbactam. Fonte: Autores (2021). 


\section{Discussão}

Os microrganismos mais prevalentes, em aspirados traqueais, foram A. baumannii, K. pneumoniae, P. auriginosa e $S$. aureus. Em um estudo desenvolvido em Bandeirantes, Paraná, foi encontrado maior número de Staphylococcus coagulase negativa (40\%), Pseudomonas (25\%) e E. coli (15\%), diferentemente do presente estudo.

A Acinetobacter baumannii é uma bacteria gram-negativa frequentemente associada às IRAS. Infelizmente, tem que se identificado cada vez mais membros do gênero Acinetobacter como causadores de infecções: Acinetobacter nosocomialis, Acinetobacter pittii, Acinetobacter dijkshoorniae e Acinetobacter seifertii (Harding et al., 2018); apesar da A. baumannii ainda ser responsável por 80\% dos casos (World Health Organization, 2019). Além disso, esse gênero causa preocupação em todos os campos de saúde pelo seu rápido desenvolvimento de mecanismos de resistência aos antibióticos (Harding et al., 2018). A prova disso é que neste estudo, esse microrganismo, apresentou altas taxas de resistência a todos os antibióticos testados, com exceção da colistina e polimixina B. Essa multirresistência é encontrada em diversos estudos (Gusatti et al., 2009; Harding et al., 2018; Ricas et al., 2013), como em pesquisa realizada no Hospital Universitário de Cuiabá, Mato Grosso, onde 85,3\% das amostras avaliadas apresentaram multirresistência, principalmente a cefalosporinas ( $3^{\circ}$ e $4^{\circ}$ geração), aminoglicosídeos e carbapenêmicos; no entanto, assim como no presente estudo, 100\% das cepas foram sensíveis a polimixina B (Ricas et al., 2013). A resistência aos carbapenêmicos pode ser explicada pela diminuição da permeabilidade das membranas externas, alteração na afinidade das proteínas ligadoras de penicilinas (PBPs) e, também, pela hiperexpressão de bombas de efluxo (Gusatti et al., 2009; Ribeiro et al., 2019). Entretanto, as principais formas de resistência aos carbapenêmicos são a expressão de metalo- $\beta$-lactamases (MBLs), as quais não são hidrolisadas por inibidores comerciais de serino $\beta$-lactamases e a expressão de oxacilinases (OXA) (Gusatti et al., 2009; Walsh et al., 2005). Os fatores de virulência que contribuem para a patogênese da A. baumannii são: adesinas e glicoconjugados de superfície, sistemas de secreção e de aquisição de micronutrientes; destacase, ainda, sua capacidade em resistir à dessecação e formar biofilmes, favorecendo seu sucesso como paógeno nosocomial (Harding et al., 2018).

A resistência da Klebsiella spp. correlaciona-se com o uso inadequado de antimicrobianos e a produção de betalactamases tipo AmpC e de carbapenemases, como as metalo-betalactamases (MBL) e carbapenemasesdo tipo KPC (Bastos et al., 2020; Marchaim et al., 2008). Além disso, são mais acometidos pacientes imunocomprometidos hospitalizados ou pacientes em uso de dispositivos invasivos pelas infecções associadas à K. pneumoniae, produtora da enzima KPC; dessa forma, a prevenção da infecção é a arma mais eficaz no combate desta bactéria (Marchaim et al., 2008). Nos aspirados traqueais coletados, a K. pneumoniae foi 100\% sensível apenas à amicacina e apresentou elevados índices de resistência aos demais antibióticos testados. Estudo realizado em Minas Gerais, Brasil, demonstrou que 36\% dos casos de infecção foram por K. pneumoniae produtora de Betalactamases de Espectro Estendido (Extended Spectrum Beta-lactamases-ESBL) e 28\% casos de K. pneumoniae produtora de carbapenemase (KPC) (Muniz et al., 2019), o que gera preocupação frente à realidade de multirresistência aos antimicrobianos por esse gênero.

A bacteria gram-negativa Pseudomonas aeruginosa é um importante patógeno oportunista que está relacionado sobretudo às infecções nosocomiais e infecções do trato respiratório (Lopes et al., 2020). Os fatores de risco para a infecção por essa bacteria são ventilação mecânica, procedimentos invasivos e tratamento inadequado, como o uso de carbapenem para seu tratamento; além de fatores como tempo de internação, comorbidades e uso de imunossupressores (Lopes et al., 2020). Neste estudo, a $P$. aeruginosa apresentou completa sensibilidade aos antimicrobianos. A resistência associada à essa bacteria está relacionada a produção de betalactamases, hiperexpressão de sistemas de efluxo, alteração da permeabilidade da membrana e pela síntese de proteínas de ligação à penicilina (PBPs) com baixa afinidade por betalactâmicos (Fuentefria, 2009). Outro importante mecanismo é sua alta capacidade de ficar aderida e sobreviver em equipamentos médicos e outras superfícies hospitalares (Dresch et al., 2018). 
O S. aureus também é um importante microrganismo relacionados às infecções nosocomias e frequentemente encontrado em diferentes superfícies (Dresch et al., 2018). Esse microrganismo coloniza cerca de 30\% das pessoas, sendo uma importante causa de bacteremia, endocardite infecciosa, infecção osteoarticular e de tecidos moles e pele, assim como infecções relacionadas a dispositivos (Tong et al., 2015). Tem-se observado um crescimento que varia de $1 \%$ (na Holanda) até 50\% (em países da América e Europa) de cepas de S. aureus resistentes à meticilina (MRSA) (Loureiro et al., 2016). Neste estudo, foi observado $20 \%$ de resistência à oxacilina.

Em relação às uroculturas, destacou-se a prevalência da bactéria E. coli (40\%), seguido pelo E. cloacae (20\%). Esse dado encontra-se em consonância com outros estudos, onde destaca-se a alta prevalência da E. coli nas culturas. Foi encontrado predominância $(53,8 \%)$ dessa bactéria em um estudo realizado em Santarém, Pará, e em uma pesquisa realizada em Cuverlo, Minas Gerais, onde a E. coli correspondeu a 67,4\% dos casos (Tiago et al., 2020; Rocha \& Resende, 2017). Essa alta prevalência ocorre porque a $E$. coli faz parte da microbiota do trato gastrointestinal humano; dessa forma, devido sua proximidade com o trato urinário, esse microrganismo torna-se um potencial causador de infecções desse sítio. Além disso, o uso de dispositivos invasivos, como sonda vesical, é um fator de risco para o desenvolvimento dessas infecções. Destaca-se ainda os fatores de virulência que incluem adesinas, toxinas, polissacarídeos, invasinas e proteases que tornam a bactéria capaz de ligar-se e lesar células e tecidos do hospedeiro fora do trato intestinal, sendo transmitida por falta de higienização, acometendo sobretudo pacientes em uso de cateter urinário (Tiago et al., 2020).

Ainda em relação a $E$. coli, destacou-se a resistência a ampicilina, cefazolina, cefepime, ceftriazona, ciprofloxacino e levofloxacino; porém, $100 \%$ de sensibilidade ao sulfametoxazol+trimetoprima pelas cepas de $E$. coli testadas. Isso contraria o que as evidências têm demonstrado, onde tem se observado aumento progressivo da resistência a sulfametoxazol+trimetoprima (Bastos et al., 2020; Rocha \& Resende, 2017; Tiago et al., 2020).

$\mathrm{O} E$. cloacae destacou-se pela resistência às penicilinas e às cefalosporinas, assim como tem se observado na literatura uma frequência considerável de resistência múltipla aos antimicrobianos por essa bacteria (Zanguña et al., 2020). Isso está relacionado a presença de pelo menos um gene associado à expressão das bombas expulsoras, sendo frequentes o MexC e o AcrB; assim como a presença das enzimas ESBL e AmpC (Bastos et al., 2020; Zanguña et al., 2020).

\section{Conclusão}

A. baumannii apresentou $100 \%$ de sensibilidade à colistina e polimixina B nos aspirados traqueias, assim como a $K$. pneumoniae apresentou à amicacina em todas as amostras coletadas. A E. coli demonstrou certa restência às cefalospororinas, com exceção da cefoxitina; além disso teve sensibilidade parcial ao sulfametoxazol+trimetoprima. Assim, a etiologia e o perfil de sensibilidade estão em consonância com os encontrados na literatura recente, tornando esse tema uma grande preocupação mundial frente aos meios adaptivados encontrados por essas bactérias multirresistentes, principalmente as produtoras de ESBL, os MRSA e a produção de carbapenemase.

Estudos como este são essenciais para o monitoramento das taxas de resistência hospitalar e para a construção, juntamente com a comissão de controle de infecção hospitalar, de condutas de antibioticoterapia empírica que visem, a diminuição da resistência bacteriana, das taxas de mortalidade, do tempo de internação e dos custos em saúde; e, assim, no controle e prevenção dessas infecções.

Pesquisas futuras analisando outros setores podem ser necessárias para a construção do conhecimento a respeito das IRAS nos serviços hospitalares, possibilitando um melhor controle dessas infecções. 


\section{Referências}

Agência Nacional de Vigilância Sanitária. (2016). Programa nacional de prevenção e controle de infecções relacionadas à assistência à saúde (2016-2020).

Agência Nacional de Vigilância Sanitária. (2017). Medidas de Prevenção de Infecção Relacionada à Assistência à Saúde.

Andrade, C. W. Q., Silva, K. S. B., Santana, M. M. R., de Oliveira, A. V., Guimarães, M. D., \& Naue, C. R. (2021). Etiologia e resistência de isolados bacterianos de hemoculturas da Sala de Cuidados Intermediários de um Hospital Universitário em Pernambuco. Research, Society and Development, 10(7).

Bastos, I. D. M., Bastos, B. D. M., Silva, C. F., Silva, K. S. B., \& Naue, C. R. (2020). Perfil bacteriano de amostras microbiológicas de pacientes internados na Clínica Cirúrgica de um Hospital Universitário de Pernambuco. VITTALLE-Revista de Ciências da Saúde, 32(1), $108-121$.

Bastos, I. D. M., Bastos, B. D. M., Silva, K. S. B., SILVA, C. F. E., \& Naue, C. R. (2020). Perfil bacteriano de amostras biológicas da clínica médica de um Hospital Universitário do Sertão de Pernambuco. Revista de Ensino, Ciência e Inovação em Saúde, 1(1), 4-15.

de Sousa, M. A. S., Nascimento, G. C., Bim, F. L., de Oliveira, L. B., \& da Silva Oliveira, A. D. (2017). Infecções hospitalares relacionadas a procedimentos invasivos em unidades de terapia intensiva: Revisão integrativa. Revista Prevenção de Infecção e Saúde, 3(3).

Dresch, F., de Freitas Birkheuer, C., Rempel, C., \& Maciel, M. J. (2018). Contaminação de superfícies localizadas em unidades de terapia intensiva e salas de cirurgia: uma revisão sistemática da literatura. Revista de Epidemiologia e Controle de Infecção, 8(1), 85-91.

Fuentefria, D. B. (2009). Detecção de metalo beta-lactamases e similaridade genética em isolados de Pseudomonas aeruginosa de efluente hospitalar e água superficial (Tese de doutorado). Universidade Federal do Rio Grande do Sul. Instituto de Ciência Básicas da Saúde. Programa de Pós-Graduação em Microbiologia e do Ambiente. Porto Alegre, RS, Brasil.

Gomes, M. F., \& Moraes, V. L. (2018). O programa de controle de infecção relacionada à assistência à saúde em meio ambiente hospitalar e o dever de fiscalização da Agência Nacional de Vigilância Sanitária. Revista de Direito Sanitário, 18(3), 43-61.

Gusatti, C. D. S., Ferreira, A. E., Fuentefria, D. B., \& Corção, G. (2009). Resistência a $\beta$-lactâmicos em Acinetobacter spp isolados de efluente hospitalar no sul do Brasil. Revista da Sociedade Brasileira de Medicina Tropical, 42, 183-187.

Harding, C. M., Hennon, S. W., \& Feldman, M. F. (2018). Uncovering the mechanisms of Acinetobacter baumannii virulence. Nature Reviews Microbiology, 16(2), 91-102.

Lopes, A. C. C., da Silva, C. A. L., de Oliveira, J. S., \& Alves, J. T. C. (2020). Fatores de risco para infecção por Pseudomonas aeruginosa em relação à infecções hospitalares. Brazilian Journal of Development, 6(1), 2121-2130.

Loureiro, R. J., Roque, F., Rodrigues, A. T., Herdeiro, M. T., \& Ramalheira, E. (2016). O uso de antibióticos e as resistências bacterianas: breves notas sobre a sua evolução. Revista Portuguesa de saúde pública, 34(1), 77-84.

Marchaim, D., Navon-Venezia, S., Schwaber, M. J., \& Carmeli, Y. (2008). Isolation of imipenem-resistant Enterobacter species: emergence of KPC-2 carbapenemase, molecular characterization, epidemiology, and outcomes. Antimicrobial agents and chemotherapy, 52(4), 1413-1418.

Muniz, J. J., Silva, A. G., Rosa, A. D. F. N., de Melo, F. A., \& Mattozo, J. M. A. (2019). Resistência aos antibióticos utilizados para tratamento de infecções por Klebsiella pneumoniae em um hospital. Revista de Ciências da Saúde Básica e Aplicada, 2, 3-10.

Oliveira, L. S., \& Santos, T. B. D. (2020). Perfil etiológico e de suscetibilidade bacteriana aos agentes antimicrobianos em isolados de hemoculturas: um levantamento bibliográfico (Trabalho de conclusão de curso). Centro Universitário Tiradentes - UNIT, Maceió, AL, Brasil.

Ribeiro, T. D. S., Ribeiro, R. A. A. D. S., Batista, K. S., Aquino, S. R. D., \& Naue, C. R. (2019). Ocorrência e perfil bacteriano de culturas coletadas em pacientes internados na unidade de terapia intensiva em um hospital terciário. HU rev, 122-133.

Ricas, R. V., Marques, T. C., \& Yamamoto, A. C. A. (2013). Perfil de resistência de Acinetobacter baumannii a antimicrobianos em um hospital universitário de Cuiabá-MT. Infarma Ciências Farmacêuticas, 25(4), 178-181.

Rocha, T. B., \& Resende, F. A. (2017). Perfil de resistência da bactéria Escherichia coli a antibióticos em infecções do trato urinário em um laboratório de Curvelo/MG. Revista Brasileira de Ciências da Vida, 5(5).

Secretaria Estadual de Saúde de Pernambuco. (2020). http://portal.saude.pe.gov.br/unidades-de-saude-e-servicos/secretaria-executiva-de-coordenacaogeral/viii-geres

Tiago, K. P. (2020). Frequência e resistência de uroculturas provenientes de pacientes internados na unidade de terapia intensiva do hospital municipal de Santarém-PA. RBAC, 52(1), 64-70.

Tong, S. Y., Davis, J. S., Eichenberger, E., Holland, T. L., \& Fowler Jr, V. G. (2015). Staphylococcus aureus infections: epidemiology, pathophysiology, clinical manifestations, and management. Clinical microbiology reviews, 28(3), 603-661.

Walsh, T. R., Toleman, M. A., Poirel, L., \& Nordmann, P. (2005). Metallo- $\beta$-lactamases: the quiet before the storm?. Clinical microbiology reviews, 18(2), 306-325.

World Health Organization. (2019). Healthcare-associated Infections: Diseases and Organisms. https://www.cdc.gov/hai/organisms/organisms.html

Zanguña-Fonseca, L. F., Torres-Caycedo, M. I., \& Di-Filippo-Iriarte, G. (2020). Perfil de tolerancia al triclosán y detección de los genes MexA, MexC, AcrB y oqxA relacionados con la expresión de bombas de expulsión en aislados clínicos del género Enterobacter aerogenes y Enterobacter cloacae. Rev. Investig. Salud. Univ. Boyacá, 102-117. 